Supporting Information for:

\title{
A New Entry to Asymmetric Synthesis of Optically Active $\alpha, \gamma$ - Substituted $\gamma$-Butyrolactones Using a Carbohydrate Derived Amide as both a Chiral Auxiliary and a Proton Source
}

Ling-Lin Huang, Ming-Hua Xu* and Guo-Qiang Lin

Shanghai Institute of Organic Chemistry, Chinese Academy of Sciences. 354 Fenglin Lu, Shanghai 200032, and Graduate School of the Chinese Academy of Sciences, China

xumh@mail.sioc.ac.cn

\section{Table of Contents}

1. Characterization data of optically active $\gamma$-butyrolactone products 5-12. $\mathrm{S} 2-\mathrm{S} 4$

2. Characterization data of new chiral methacrylate compounds $\mathbf{3}$ and $\mathbf{4}$.

S4 - S6

3. Supposed reaction transition state model. 


\section{Characterization data of optically active $\gamma$-butyrolactone products 5-10:}

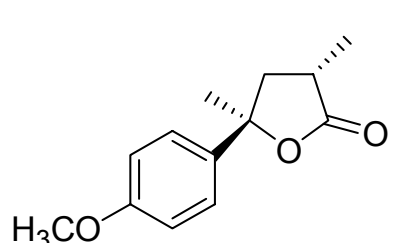

trans-5. $[\alpha]_{\mathrm{D}}{ }^{20}+14.0\left(\mathrm{c} 0.06, \mathrm{CHCl}_{3}\right)$ for $99 \%$ ee; ${ }^{1} \mathrm{H}$ NMR $(300$ $\left.\mathrm{MHz}, \mathrm{CDCl}_{3}\right): \delta 1.25(\mathrm{~d}, 3 \mathrm{H}, J=6.9 \mathrm{~Hz}), 1.71(\mathrm{~s}, 3 \mathrm{H}), 2.00(\mathrm{t}$, $1 \mathrm{H}, J=11.7 \mathrm{~Hz}), 2.48-2.57(\mathrm{~m}, 1 \mathrm{H}), 2.71-2.78(\mathrm{~m}, 1 \mathrm{H}), 3.80(\mathrm{~s}$, $3 \mathrm{H}), 6.88\left(\mathrm{dd}, 2 \mathrm{H}, J_{l}=6.6 \mathrm{~Hz}, J_{2}=4.2 \mathrm{~Hz}\right), 7.28\left(\mathrm{dd}, 2 \mathrm{H}, J_{l}=\right.$ $\left.6.9 \mathrm{~Hz}, J_{2}=4.8 \mathrm{~Hz}\right) ;{ }^{13} \mathrm{C} \mathrm{NMR}\left(75 \mathrm{MHz}, \mathrm{CDCl}_{3}\right) \delta 14.6,30.4$, $35.0,44.8,55.2,84.4,113.8,125.4,135.8,158.9,179.3$.

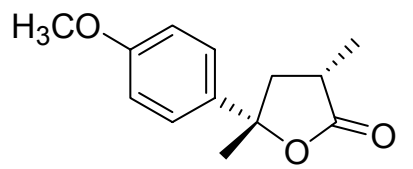

cis-5. $[\alpha]_{\mathrm{D}}{ }^{20}+10.0\left(\mathrm{c} 0.11, \mathrm{CHCl}_{3}\right)$ for $99 \%$ ee; ${ }^{1} \mathrm{H}$ NMR $(300$ $\left.\mathrm{MHz}, \mathrm{CDCl}_{3}\right): \delta 1.26(\mathrm{~d}, 3 \mathrm{H}, J=6.9 \mathrm{~Hz}), 1.65(\mathrm{~s}, 3 \mathrm{H}), 2.08(\mathrm{t}$, $1 \mathrm{H}, J=11.4 \mathrm{~Hz}), 2.63-2.71(\mathrm{~m}, 1 \mathrm{H}), 2.89-2.98(\mathrm{~m}, 1 \mathrm{H}), 3.81(\mathrm{~s}$, $3 \mathrm{H}), 6.90\left(\mathrm{dd}, 2 \mathrm{H}, J_{l}=6.6 \mathrm{~Hz}, J_{2}=2.1 \mathrm{~Hz}\right), 7.29\left(\mathrm{dd}, 2 \mathrm{H}, J_{l}=\right.$ $\left.6.9 \mathrm{~Hz}, J_{2}=2.1 \mathrm{~Hz}\right) ;{ }^{13} \mathrm{C} \mathrm{NMR}\left(75 \mathrm{MHz}, \mathrm{CDCl}_{3}\right) \delta 15.4,28.8,35.3,44.0,55.3,84.4$, $113.8,125.2,137.4,158.8,178.9$.

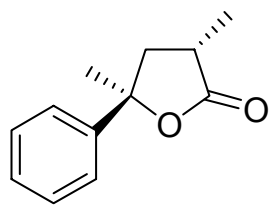

trans-6. $[\alpha]_{\mathrm{D}}{ }^{20}+32.0\left(\mathrm{c} 0.35, \mathrm{CHCl}_{3}\right)$ for $97 \% e e ;{ }^{1} \mathrm{H}$ NMR $(300 \mathrm{MHz}$, $\left.\mathrm{CDCl}_{3}\right): \delta 1.17(\mathrm{~d}, 3 \mathrm{H}, J=6.9 \mathrm{~Hz}), 1.59(\mathrm{~s}, 3 \mathrm{H}), 2.01(\mathrm{t}, 1 \mathrm{H}, J=10.8$ $\mathrm{Hz}), 2.59-2.66(\mathrm{~m}, 1 \mathrm{H}), 2.82-2.91(\mathrm{~m}, 1 \mathrm{H}), 7.18-7.32(\mathrm{~m}, 5 \mathrm{H}) ;{ }^{13} \mathrm{C}$ NMR $\left(75 \mathrm{MHz}, \mathrm{CDCl}_{3}\right) \delta 15.4,28.8,35.2,43.9,84.4,123.9,127.5$, $128.5,145.3,178.9$.

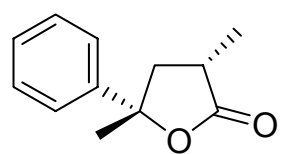

cis-6. ${ }^{1} \mathrm{H}$ NMR $\left(300 \mathrm{MHz}, \mathrm{CDCl}_{3}\right): \delta 1.24(\mathrm{~d}, 3 \mathrm{H}, J=7.2 \mathrm{~Hz}), 1.71(\mathrm{~s}$, $3 \mathrm{H}), 2.08(\mathrm{t}, 1 \mathrm{H}, J=11.4 \mathrm{~Hz}), 2.67-2.74(\mathrm{~m}, 1 \mathrm{H}), 2.89-2.98(\mathrm{~m}, 1 \mathrm{H})$, 7.26-7.40 (m, 5H) ; ${ }^{13} \mathrm{C} \mathrm{NMR}\left(75 \mathrm{MHz}, \mathrm{CDCl}_{3}\right) \delta 15.3,28.7,35.2$, $43.9,84.4,123.8,127.5,128.5,145.3,178.8$.

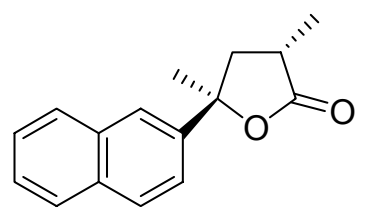

trans-7. $[\alpha]_{\mathrm{D}}{ }^{20}-6.1\left(\mathrm{c} 0.77, \mathrm{CHCl}_{3}\right)$ for $96 \% e e ;{ }^{1} \mathrm{H}$ NMR $(300$ $\left.\mathrm{MHz}, \mathrm{CDCl}_{3}\right): \delta 1.26(\mathrm{~d}, 3 \mathrm{H}, J=10.2 \mathrm{~Hz}), 1.80(\mathrm{~s}, 3 \mathrm{H}), 2.09(\mathrm{t}$, $1 \mathrm{H}, J=12.9 \mathrm{~Hz}), 2.52-2.56(\mathrm{~m}, 1 \mathrm{H}), 2.83-2.80(\mathrm{~m}, 1 \mathrm{H}), 7.39-7.50$ (m, 3H), 7.80-7.86 (m, 4H); ${ }^{13} \mathrm{C}$ NMR $\left(75 \mathrm{MHz}, \mathrm{CDCl}_{3}\right) \delta 14.6$, $30.1,34.9,44.7,84.6,122.5,122.7,126.3,126.6,127.5,128.1$, $128.6,132.5,132.9,140.9,179.3$.

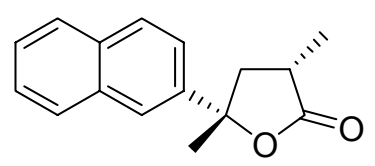

cis-7. ${ }^{1} \mathrm{H}$ NMR $\left(300 \mathrm{MHz}, \mathrm{CDCl}_{3}\right): \delta 1.27(\mathrm{~d}, 3 \mathrm{H}, J=7.2 \mathrm{~Hz})$, $1.76(\mathrm{~s}, 3 \mathrm{H}), 2.19(\mathrm{~m}, 1 \mathrm{H}, J=10.8 \mathrm{~Hz}), 2.75-2.82(\mathrm{~m}, 1 \mathrm{H}), 2.92-$ $3.03(\mathrm{~m}, 1 \mathrm{H}), 7.43-7.53(\mathrm{~m}, 3 \mathrm{H}), 7.82-7.90(\mathrm{~m}, 4 \mathrm{H}) ;{ }^{13} \mathrm{C}$ NMR $\left(75 \mathrm{MHz}, \mathrm{CDCl}_{3}\right) \delta 15.4,28.6,35.2,43.8,84.5,122.2,122.3$, $126.2,126.4,127.5,128.1,128.5,132.4,132.9,142.5,178.9$. 


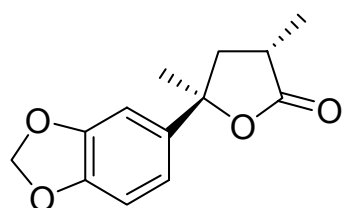

179.1 .

trans-8. $[\alpha]_{\mathrm{D}}{ }^{20}+24.4\left(\mathrm{c} 1.42, \mathrm{CHCl}_{3}\right)$ for $96 \%$ ee; ${ }^{1} \mathrm{H}$ NMR $(300$ $\left.\mathrm{MHz}, \mathrm{CDCl}_{3}\right): \delta 1.24(\mathrm{~d}, 3 \mathrm{H}, J=7.2 \mathrm{~Hz}), 1.70(\mathrm{~s}, 3 \mathrm{H}), 1.98(\mathrm{t}, 1 \mathrm{H}$, $J=12.0 \mathrm{~Hz}), 2.47-2.57(\mathrm{~m}, 1 \mathrm{H}), 2.67-2.74(\mathrm{~m}, 1 \mathrm{H}), 5.95(\mathrm{~s}, 2 \mathrm{H})$, 6.74-6.83 (m, 3H) ; ${ }^{13} \mathrm{C} \mathrm{NMR}\left(75 \mathrm{MHz}, \mathrm{CDCl}_{3}\right) \delta$ 14.6, 30.1, 35.0, 44.9, 84.4, 101.2, 105.3, 108.0, 117.3, 137.8, 146.9, 147.9,

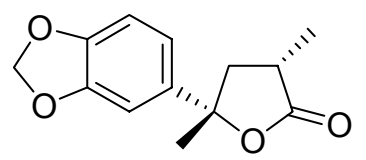

cis-8. ${ }^{1} \mathrm{H}$ NMR $\left(300 \mathrm{MHz}, \mathrm{CDCl}_{3}\right): \delta 1.26(\mathrm{~d}, 3 \mathrm{H}, J=7.2 \mathrm{~Hz})$, $1.64(\mathrm{~s}, 3 \mathrm{H}), 2.08(\mathrm{t}, 1 \mathrm{H}, J=10.8 \mathrm{~Hz}), 2.62-2.69(\mathrm{~m}, 1 \mathrm{H}), 2.88-$ $2.97(\mathrm{~m}, 1 \mathrm{H}), 5.97(\mathrm{~s}, 2 \mathrm{H}), 6.77-6.89(\mathrm{~m}, 3 \mathrm{H}) ;{ }^{13} \mathrm{C} \mathrm{NMR}(75 \mathrm{MHz}$, $\left.\mathrm{CDCl}_{3}\right) \delta 15.4,28.8,35.3,44.1,84.3,101.1,105.1,108.1,117.1$, $139.3,146.8,147.7,178.7$.<smiles>C[C@H]1C[C@](C)(c2ccc(Br)cc2)OC1=O</smiles>

trans-9. $[\alpha]_{\mathrm{D}}{ }^{20}+9.8\left(\mathrm{c} 1.05, \mathrm{CHCl}_{3}\right)$ for $96 \% e e ;{ }^{1} \mathrm{H}$ NMR $(300$ $\left.\mathrm{MHz}, \mathrm{CDCl}_{3}\right): \delta 1.25(\mathrm{~d}, 3 \mathrm{H}, J=6.9 \mathrm{~Hz}), 1.71(\mathrm{~s}, 3 \mathrm{H}), 2.03(\mathrm{t}, 1 \mathrm{H}$, $J=11.7 \mathrm{~Hz}), 2.45-2.54(\mathrm{~m}, 1 \mathrm{H}), 2.68-2.75(\mathrm{~m}, 1 \mathrm{H}), 7.25(\mathrm{~d}, 2 \mathrm{H}, J$ $=9.0 \mathrm{~Hz}), 7.50(\mathrm{~d}, 2 \mathrm{H}, J=8.7 \mathrm{~Hz}) ;{ }^{13} \mathrm{C} \mathrm{NMR}\left(75 \mathrm{MHz}, \mathrm{CDCl}_{3}\right) \delta$ $14.7,30.2,34.9,44.8,84.0,121.6,126.1,131.7,142.9,178.9$.<smiles>C[C@H]1C[C@](C)(c2ccc(Br)cc2)OC1=O</smiles>

cis-9. ${ }^{1} \mathrm{H}$ NMR $\left(300 \mathrm{MHz}, \mathrm{CDCl}_{3}\right): \delta 1.25(\mathrm{~d}, 3 \mathrm{H}, J=6.9 \mathrm{~Hz}), 1.64$ $(\mathrm{s}, 3 \mathrm{H}), 2.03(\mathrm{t}, 1 \mathrm{H}, J=12.9 \mathrm{~Hz}), 2.65-2.72(\mathrm{~m}, 1 \mathrm{H}), 2.89-2.98(\mathrm{~m}$, $1 \mathrm{H}), 7.23-7.28(\mathrm{~m}, 2 \mathrm{H}), 7.47-7.52(\mathrm{~m}, 2 \mathrm{H}) ;{ }^{13} \mathrm{C} \mathrm{NMR}(75 \mathrm{MHz}$, $\left.\mathrm{CDCl}_{3}\right) \delta 15.4,28.7,35.2,43.8,83.9,121.5,125.7,131.7,144.4$,

178.5 .

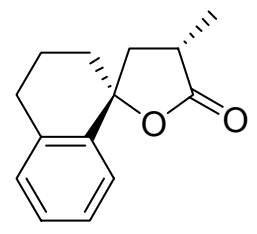

trans-10. ${ }^{1} \mathrm{H}$ NMR $\left(300 \mathrm{MHz}, \mathrm{CDCl}_{3}\right): \delta 1.36(\mathrm{~d}, 3 \mathrm{H}, J=7.7 \mathrm{~Hz}), 1.92-$ $2.05(\mathrm{~m}, 4 \mathrm{H}), 2.18-2.26(\mathrm{~m}, 1 \mathrm{H}), 2.66-2.73(\mathrm{~m}, 1 \mathrm{H}), 2.82-2.88(\mathrm{~m}, 2 \mathrm{H})$, 3.02-3.11 (m, 1H), 7.10-7.26 (m, 4H); ${ }^{13} \mathrm{C} \mathrm{NMR}\left(75 \mathrm{MHz}, \mathrm{CDCl}_{3}\right) \delta$ $16.7,19.2,28.5,35.1,37.9,44.7,83.4,124.7,126.3,127.9,129.3,136.4$, $138.8,178.8$.

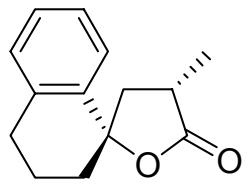

cis-10. $[\alpha]_{\mathrm{D}}{ }^{20}+20.0\left(\mathrm{c} 0.16, \mathrm{CHCl}_{3}\right)$ for $90 \%$ ee; ${ }^{1} \mathrm{H}$ NMR $(300 \mathrm{MHz}$, $\left.\mathrm{CDCl}_{3}\right): \delta 1.37(\mathrm{~d}, 3 \mathrm{H}, J=7.2 \mathrm{~Hz}), 1.81-1.89(\mathrm{~m}, 1 \mathrm{H}), 1.99-2.10(\mathrm{~m}, 4 \mathrm{H})$, 2.63-2.71 (m, 1H), 2.81-2.98 (m, 3H), 7.09-7.37 (m, 4H); ${ }^{13} \mathrm{C}$ NMR $(75$ $127.9,128.7,137.3,138.3,178.3$. 


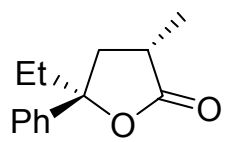

trans-11. ${ }^{1} \mathrm{H}$ NMR $\left(300 \mathrm{MHz}, \mathrm{CDCl}_{3}\right): \delta 0.82(\mathrm{t}, 3 \mathrm{H}, J=7.5 \mathrm{~Hz}), 1.25(\mathrm{~d}$, $3 \mathrm{H}, J=7.2 \mathrm{~Hz}), 1.94-2.01(\mathrm{~m}, 3 \mathrm{H}), 2.46-2.55(\mathrm{~m}, 1 \mathrm{H}), 2.72\left(\mathrm{dd}, 1 \mathrm{H}, J_{l}=\right.$ $\left.12.3 \mathrm{~Hz}, J_{2}=8.4 \mathrm{~Hz}\right), 7.28-7.41(\mathrm{~m}, 5 \mathrm{H}){ }^{13} \mathrm{C} \mathrm{NMR}\left(75 \mathrm{MHz}, \mathrm{CDCl}_{3}\right) \delta$ $8.23,14.67,34.60,35.63,43.33,87.40,124.90,127.49$, 128.43, 142.13, 179.37.

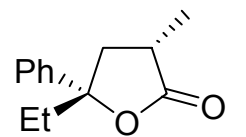

cis-11. ${ }^{1} \mathrm{H}$ NMR $\left(300 \mathrm{MHz}, \mathrm{CDCl}_{3}\right): \delta 0.82(\mathrm{t}, 3 \mathrm{H}, J=7.5 \mathrm{~Hz}), 1.25(\mathrm{~d}$, $3 \mathrm{H}, J=6.9 \mathrm{~Hz}), 1.91-2.13(\mathrm{~m}, 3 \mathrm{H}), 2,78\left(\mathrm{dd}, 1 \mathrm{H}, J_{1}=12.3 \mathrm{~Hz}, J_{2}=9 \mathrm{~Hz}\right)$, 2.87-2.92 (m, 1H), 7.26-7.41 (m.5H) ${ }^{13} \mathrm{C}$ NMR $\left(75 \mathrm{MHz}, \mathrm{CDCl}_{3}\right) \delta 8.22$, $15.78,34.69,35.14,42.57,87.33,124.44,127.29,128.33,143.69,179.15$.

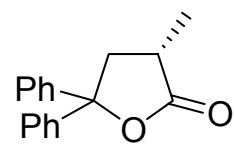

12. ${ }^{1} \mathrm{H}$ NMR $\left(300 \mathrm{MHz}, \mathrm{CDCl}_{3}\right): \delta 1.29(\mathrm{~d}, 3 \mathrm{H}, J=7.0), 2.47(\mathrm{t}, 1 \mathrm{H}, J=$ $12.0 \mathrm{~Hz}), 2.63-2.72(\mathrm{~m}, 1 \mathrm{H}), 3.23\left(\mathrm{dd}, 1 \mathrm{H}, J_{1}=12.2 \mathrm{~Hz}, J_{2}=7.6 \mathrm{~Hz}\right)$, 7.25-7.47 (m, 10H). ${ }^{13} \mathrm{C}$ NMR $\left(75 \mathrm{MHz}, \mathrm{CDCl}_{3}\right) \delta 14.58,35.09,43.83$, $87.19,125.23,125.27,127.77,127.79,128.49,128.61,142.54,143.81,178.68$.

\section{Characterization data of new chiral methacrylate compounds 3 and 4:}

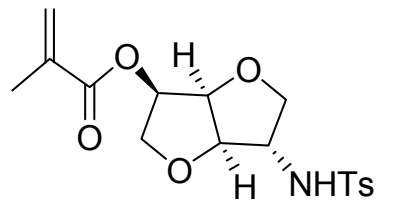

3a. $[\alpha]_{\mathrm{D}}{ }^{20}+45.8\left(\mathrm{c} 0.39, \mathrm{CHCl}_{3}\right) ;{ }^{1} \mathrm{H} \mathrm{NMR}\left(300 \mathrm{MHz}, \mathrm{CDCl}_{3}\right)$ : $\delta 1.94(\mathrm{~s}, 3 \mathrm{H}), 2.44(\mathrm{~s}, 3 \mathrm{H}), 3.60-3.90(\mathrm{~m}, 5 \mathrm{H}), 4.37$ (d, $1 \mathrm{H}, J=$ $4.5 \mathrm{~Hz}), 4.79(\mathrm{t}, 3 \mathrm{H}, \quad J=5.4 \mathrm{~Hz}), 4.96-5.18(\mathrm{~m}, 2 \mathrm{H}), 5.60(\mathrm{t}, 1 \mathrm{H}$, $7.2 \mathrm{~Hz}) ;{ }^{1} \mathrm{C}$ NMR $\left(75 \mathrm{MHz}, \mathrm{CDCl}_{3}\right) \delta 18.2,21.5,59.3,71.1,73.0,73.9,80.5,87.1$ 126.3, 127.0, 129.9, 135.5, 137.0, 143.9, 166.6; FT-IR (KBr) v 3265, 2926, 1721, 1338 $\mathrm{cm}^{-1}$; EI-MS (m/z, \%): 367(M+1, 2.99), 197(20.06), 155(31.07), 110(12.21), 91(56.62), 69(100.00), 41(32.27); HRMS (EI) calcd for $\mathrm{C}_{17} \mathrm{H}_{21} \mathrm{NO}_{6} \mathrm{~S} 367.1090$, found 367.1116 .

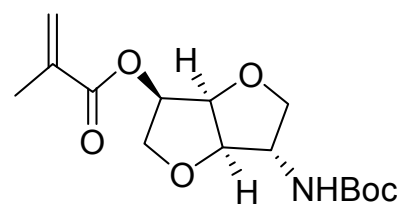

3b. $[\alpha]_{\mathrm{D}}{ }^{20}+39.1\left(\mathrm{c} 1.00, \mathrm{CHCl}_{3}\right) ;{ }^{1} \mathrm{H} \mathrm{NMR}\left(300 \mathrm{MHz}, \mathrm{CDCl}_{3}\right): \delta$ $1.29(\mathrm{~s}, 9 \mathrm{H}), 1.97(\mathrm{~s}, 3 \mathrm{H}), 3.77-3.97(\mathrm{~m}, 4 \mathrm{H}), 4.11-4.21(\mathrm{~m}, 1 \mathrm{H})$, $4.39(\mathrm{~d}, 1 \mathrm{H}, J=3.9 \mathrm{~Hz}), 4.71-4.72(\mathrm{~m}, 2 \mathrm{H}), 5.20(\mathrm{~s}, 1 \mathrm{H}), 5.62$ $(\mathrm{s}, 1 \mathrm{H}), 6.16(\mathrm{~s}, 1 \mathrm{H}) ;{ }^{13} \mathrm{C} \mathrm{NMR}\left(75 \mathrm{MHz}, \mathrm{CDCl}_{3}\right): \delta 18.3,28.4$, 57.3, 71.1, 73.2, 74.2, 80.0, 87.5, 126.2, 135.8, 155.1, 166.7; FT-IR (KBr) v 3339, 2980, 1720, $1522 \mathrm{~cm}^{-1}$; EI-MS (m/z，\%): 141(25.81), 110(26.85), 103(20.16), 86(18.61), 84(31.36), 69(100.00), 68(19.35), 57(98.34), 41(40.47); HRMS (EI) calcd for $\mathrm{C}_{15} \mathrm{H}_{23} \mathrm{O}_{6} \mathrm{~N}$ 336.1418 , found 336.1430 .

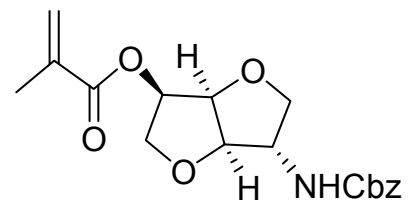

3c. $[\alpha]_{\mathrm{D}}{ }^{20}+38.6\left(c 0.40, \mathrm{CHCl}_{3}\right) ;{ }^{1} \mathrm{H} \mathrm{NMR}\left(300 \mathrm{MHz}, \mathrm{CDCl}_{3}\right)$ : $\delta 1.97(\mathrm{~s}, 3 \mathrm{H}), 3.79-3.98(\mathrm{~m}, 4 \mathrm{H}), 4.26-4.42(\mathrm{~m}, 2 \mathrm{H}), 4.80(\mathrm{t}$, $1 \mathrm{H}, J=8.1 \mathrm{~Hz}), 5.01-5.23(\mathrm{~m}, 4 \mathrm{H}), 5.63(\mathrm{~s}, 1 \mathrm{H}), 6.16(\mathrm{~s}, 1 \mathrm{H})$, $7.33(\mathrm{~s}, 5 \mathrm{H}) ;{ }^{13} \mathrm{C}$ NMR $\left(75 \mathrm{MHz}, \mathrm{CDCl}_{3}\right): \delta 18.3,57.6,67.0$, 
71.2, 73.0, 74.1, 80.5, 87.2, 126.3, 128.2, 128.3, 128.6, 135.7, 136.2, 155.6, 166.7; EI-MS (m/z, \%): 231(10.06), 158(12.04), 108(7.27), 107(9.08), 91(100.00), 69(37.41), 41(14.93); HRMS (EI) calcd for $\mathrm{C}_{18} \mathrm{H}_{21} \mathrm{NO} 347.1369$, found 347.1366 .

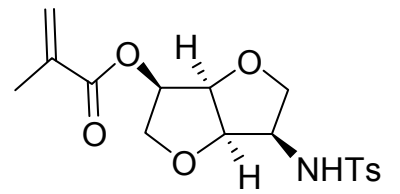

4a. $[\alpha]_{\mathrm{D}}{ }^{20}+74.6\left(\mathrm{c} 0.27, \mathrm{CHCl}_{3}\right) ;{ }^{1} \mathrm{H} \mathrm{NMR}\left(300 \mathrm{MHz}, \mathrm{CDCl}_{3}\right): \delta$ $1.96(\mathrm{~s}, 3 \mathrm{H}), 2.45(\mathrm{~s}, 3 \mathrm{H}), 3.37(\mathrm{t}, 1 \mathrm{H}, J=9.0 \mathrm{~Hz}), 3.85-4.11(\mathrm{~m}$, $5 \mathrm{H}), 4.72(\mathrm{t}, 1 \mathrm{H}, J=6.8 \mathrm{~Hz}), 5.17-5.24(\mathrm{~m}, 2 \mathrm{H}), 5.63(\mathrm{t}, 1 \mathrm{H}, J=$ $1.5 \mathrm{~Hz}), 6.15(\mathrm{~s}, 1 \mathrm{H}), 7.32(\mathrm{~d}, 2 \mathrm{H}, J=8.1 \mathrm{~Hz}), 7.80(\mathrm{~d}, 2 \mathrm{H}, J=$ $8.7 \mathrm{~Hz}) ;{ }^{13} \mathrm{C}$ NMR $\left(75 \mathrm{MHz}, \mathrm{CDCl}_{3}\right): \delta 18.2,21.6,55.7,71.3,71.4,74.4,80.2,80.8$, 126.6, 127.1, 129.8, 135.5, 137.2, 143.8, 166.6; FT-IR (KBr) v 3263, 2981, 1718, 1336, 1165, 1091, $667 \mathrm{~cm}^{-1}$; EI-MS (m/z, \%): 367(M+, 3.92), 281(5.21), 214(10.64), 197(22.29), 155(29.89), 133 (11.78), 110(11.40), 91(52.30), 69(100.00); HRMS (EI) calcd for $\mathrm{C}_{17} \mathrm{H}_{21} \mathrm{O}_{6} \mathrm{NS}$ 367.1090, found 367.1116.

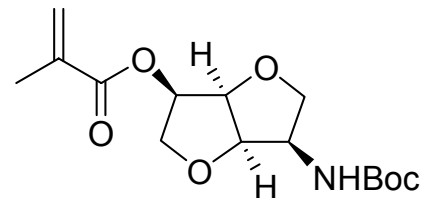

4b. $[\alpha]_{\mathrm{D}}{ }^{20}+45.2$ (c $\left.0.91, \mathrm{CHCl}_{3}\right) ;{ }^{1} \mathrm{H}$ NMR $(300 \mathrm{MHz}$, $\left.\mathrm{CDCl}_{3}\right): \delta 1.41(\mathrm{~s}, 9 \mathrm{H}), 1.97(\mathrm{~s}, 3 \mathrm{H}), 3.36(\mathrm{t}, 1 \mathrm{H}, J=7.8 \mathrm{~Hz})$, $3.89-4.17(\mathrm{~m}, 4 \mathrm{H}), 4.46(\mathrm{t}, 1 \mathrm{H}, J=4.8 \mathrm{~Hz}), 4.82(\mathrm{t}, 1 \mathrm{H}, J=5.4$

$5.7 \mathrm{~Hz}), 5.63(\mathrm{H}, 1 \mathrm{H}, J=1.5 \mathrm{~Hz}), 6.17(\mathrm{~s}, 1 \mathrm{H}),{ }^{13} \mathrm{C} \mathrm{N \textrm {Hz }}$, $5.23\left(\mathrm{dd}, 1 \mathrm{H}, J_{I}=7.8 \mathrm{~Hz}, J_{2}=\right.$ $5.7 \mathrm{~Hz}), 5.63(\mathrm{t}, 1 \mathrm{H}, J=1.5 \mathrm{~Hz}), 6.17(\mathrm{~s}, 1 \mathrm{H}) ;{ }^{13} \mathrm{C} \mathrm{NMR}\left(75 \mathrm{MHz}, \mathrm{CDCl}_{3}\right): \delta 18.3,28.4$, 53.7, 71.4, 71.6, 74.7, 81.0, 81.4, 126.3, 135.7, 155.4, 166.7; FT-IR (KBr) $\vee 3344,2980$, 2932, 1721, $1506 \mathrm{~cm}^{-1}$; ESI-MS (m/z, \%) : $314.2\left(\mathrm{M}^{+}+\mathrm{H}\right)$; HRMS (ESI) calcd for $\mathrm{C}_{15} \mathrm{H}_{23} \mathrm{O}_{6} \mathrm{NNa}\left(\mathrm{M}+\mathrm{Na}^{+}\right) 336.1418$, found 336.1423.

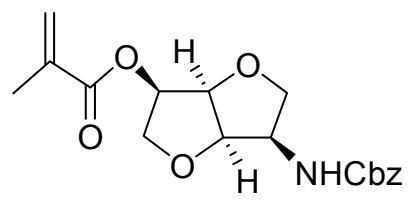

4c. $[\alpha]_{\mathrm{D}}{ }^{20}+47.0\left(\mathrm{c} 0.26, \mathrm{CHCl}_{3}\right) ;{ }^{1} \mathrm{H} \mathrm{NMR}\left(300 \mathrm{MHz}, \mathrm{CDCl}_{3}\right)$ : $\delta 1.96(\mathrm{~s}, 3 \mathrm{H}), 3.38\left(\mathrm{dd}, 1 \mathrm{H}, J_{l}=9.6 \mathrm{~Hz}, J_{2}=8.4 \mathrm{~Hz}\right), 3.88$ $4.02(\mathrm{~m}, 2 \mathrm{H}), 4.15(\mathrm{t}, 1 \mathrm{H}, J=7.8 \mathrm{~Hz}), 4.23-4.38(\mathrm{~m}, 1 \mathrm{H}), 4.46$ $(\mathrm{t}, 1 \mathrm{H}, J=5.1 \mathrm{~Hz}), 4.82(\mathrm{t}, 1 \mathrm{H}, J=5.4 \mathrm{~Hz}), 5.11(\mathrm{~s}, 2 \mathrm{H}), 5.23$ $\left(\mathrm{dd}, 1 \mathrm{H}, J_{I}=10.8 \mathrm{~Hz}, J_{2}=5.7 \mathrm{~Hz}\right), 5.36(\mathrm{~d}, 1 \mathrm{H}, J=8.1 \mathrm{~Hz}), 5.62(\mathrm{t}, 1 \mathrm{H}, J=1.5 \mathrm{~Hz}), 6.16$ $(\mathrm{s}, 1 \mathrm{H}), 7.27-7.37(\mathrm{~m}, 5 \mathrm{H}) ;{ }^{13} \mathrm{C}$ NMR $\left(75 \mathrm{MHz}, \mathrm{CDCl}_{3}\right): \delta 18.4,54.1,67.1,71.5,74.6$, 81.1, 81.3, 126.4, 128.2, 128.3, 128.6, 135.7, 136.3, 155.9, 166.7; FT-IR (KBr) v 3352, 1722, 1517, $1174 \mathrm{~cm}^{-1}$; EIMS (m/z, \%): 347( $\left.\mathrm{M}^{+}, 0.55\right), 158(30.14), 108(10.52)$, 107(11.56), 92(9.26), 91(100.00), 69(42.72), 68(9.05), 41(19.10); HRMS (MALDI) calcd for $\mathrm{C}_{18} \mathrm{H}_{21} \mathrm{O}_{6} \mathrm{~N} 348.1442$, found 348.1458 .

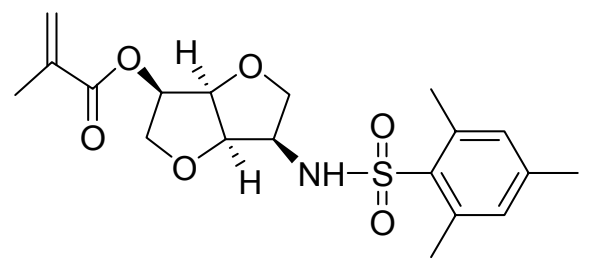

4d. $\left.[\alpha]_{\mathrm{D}}{ }^{20}+77.0(c) 0.25, \mathrm{CHCl}_{3}\right) ;{ }^{1} \mathrm{H}$ NMR $(300$ $\left.\mathrm{MHz}, \mathrm{CDCl}_{3}\right): \delta 1.96(\mathrm{~s}, 3 \mathrm{H}), 2.33(\mathrm{~s}, 3 \mathrm{H}), 2.67(\mathrm{~s}$, $6 \mathrm{H}), 3.27(\mathrm{~m}, 1 \mathrm{H}), 3.70-3.79(\mathrm{~m}, 1 \mathrm{H}), 3.87-3.99(\mathrm{~m}$, $3 \mathrm{H}), 4.13(\mathrm{t}, 1 \mathrm{H}, J=5.1 \mathrm{~Hz}), 4.73(\mathrm{t}, 1 \mathrm{H}, J=5.1 \mathrm{~Hz})$, $5.19\left(\mathrm{dd}, 1 \mathrm{H}, J_{1}=11.1 \mathrm{~Hz}, J_{2}=5.1 \mathrm{~Hz}\right), 5.31-5.34(\mathrm{~d}$, $1 \mathrm{H}, J=9.6 \mathrm{~Hz}), 5.63(\mathrm{~s}, 1 \mathrm{H}), 6.15(\mathrm{t}, 1 \mathrm{H}, J=1.2 \mathrm{~Hz})$, 
$6.98(\mathrm{~s}, 2 \mathrm{H})$; FT-IR (KBr) $v$ 661, 1159, 1331, 1712, 3339, $3363 \mathrm{~cm}^{-1}$; EI-MS (m/z, \%): $395\left(\mathrm{M}^{+}, 2.96\right), 210$ (27.05), 183 (20.98), 120 (19.27), 119 (100), 91 (24.08), 69 (97.34), 68 (18.94), 41 (30.79); Anal. Calcd for $\mathrm{C}_{19} \mathrm{H}_{25} \mathrm{O}_{6} \mathrm{NS}$ : C 57.70, H 6.37, N 3.54; Found C $57.70, \mathrm{H} 6.21, \mathrm{~N} 3.55$.

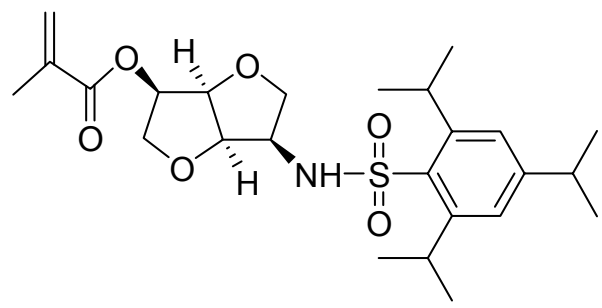

4e. $[\alpha]_{\mathrm{D}}{ }^{20}+76.0\left(\mathrm{c} 0.13, \mathrm{CHCl}_{3}\right) ;{ }^{1} \mathrm{H}$ NMR $(300$ $\left.\mathrm{MHz}, \mathrm{CDCl}_{3}\right): \delta 1.21-1.38(\mathrm{~m}, 18 \mathrm{H}), 1.94(\mathrm{~s}, 3 \mathrm{H})$, 2.58-2.93 (m, 1H), $3.34(\mathrm{t}, 1 \mathrm{H}), 3.82-3.99(\mathrm{~m}, 4 \mathrm{H})$, $4.08-4.18(\mathrm{~m}, 2 \mathrm{H}), 4.32(\mathrm{t}, 1 \mathrm{H}), 4.75(\mathrm{t}, 1 \mathrm{H}), 5.16-$ $5.22(\mathrm{~m}, 2 \mathrm{H}), 5.61(\mathrm{~s}, 1 \mathrm{H}), 6.13(\mathrm{~s}, 1 \mathrm{H}), 7.17(\mathrm{~s}$, 2H); FT-IR (KBr) v 1173, 1321, 1720, 2865, 2956, $3259 \mathrm{~cm}^{-1}$; EI-MS (m/z, \%): 394 (1.61), 267 (100.00), 251 (24.30), 218 (20.23), 213 (36.75), 187 (41.79), 69 (52.89), 43 (22.05), 41 (23.01); Anal. Calcd for $\mathrm{C}_{25} \mathrm{H}_{37} \mathrm{O}_{6} \mathrm{NS}$ : C 62.60, H 7.78, N 2.92; Found C 62.65, H 7.84, N 2.76.<smiles>C=C(C)C(=O)OC1[C@H]2OC(C)(C)O[C@H]2CO[C@]12CNC(=O)O2</smiles>

11. $[\alpha]_{\mathrm{D}}{ }^{20}-127.7\left(c 0.74, \mathrm{CHCl}_{3}\right) ;{ }^{1} \mathrm{H}$ NMR $\left(300 \mathrm{MHz}, \mathrm{CDCl}_{3}\right): \delta$ $1.38(\mathrm{~s}, 3 \mathrm{H}), 1.60(\mathrm{~s}, 3 \mathrm{H}), 2.00(\mathrm{~s}, 3 \mathrm{H}), 3.48(\mathrm{~s}, 2 \mathrm{H}), 4.20-4.35(\mathrm{~m}$, $3 \mathrm{H}), 4.44(\mathrm{~m}, 1 \mathrm{H}), 5.25(\mathrm{~d}, 1 \mathrm{H}, J=7.2 \mathrm{~Hz}), 5.67(\mathrm{t}, 2 \mathrm{H}, J=1.5 \mathrm{~Hz})$, $6.21(\mathrm{~s}, 1 \mathrm{H}) ;{ }^{13} \mathrm{C} \mathrm{NMR}\left(75 \mathrm{MHz}, \mathrm{CDCl}_{3}\right): \delta 18.3,26.4,27.8,48.6$, $61.8,71.8,73.2,74.3,103.4,110.2,127.7,135.2,156.9,166.6$; FTIR $(\mathrm{KBr}) \vee 3275,1755,1720 \mathrm{~cm}^{-1}$; EIMS (m/z, \%): $313\left(\mathrm{M}^{+}, 0.25\right)$, 298 (26.29), 85 (8.97), 70 (6.29), 69 (100.00), 59 (13.33), 43 (30.68), 41 (45.16); Anal. Calcd for $\mathrm{C}_{14} \mathrm{H}_{19} \mathrm{O}_{7} \mathrm{~N}$ : C 53.67, H 6.11, N 4.47; Found C 53.89, H 6.16, $\mathrm{N} 4.44$.<smiles>C=C(C)C(=O)O[C@H]1[C@H]2OC(C)(C)O[C@H]2CO[C@]12CNC(=O)O2</smiles>

12. $[\alpha]_{\mathrm{D}}{ }^{20}-129.0\left(c 0.13, \mathrm{CHCl}_{3}\right) ;{ }^{1} \mathrm{H}$ NMR $\left(300 \mathrm{MHz}, \mathrm{CD}_{3} \mathrm{OD}\right): \delta$ $1.31(\mathrm{~s}, 3 \mathrm{H}), 1.45(\mathrm{~s}, 3 \mathrm{H}), 1.96(\mathrm{~s}, 3 \mathrm{H}), 3.31(\mathrm{~s}, 1 \mathrm{H}), 3.42(\mathrm{~d}, 1 \mathrm{H}, J=$ $7.5 \mathrm{~Hz}), 3.80,4.01\left(\mathrm{AB}, 2 \mathrm{H}, J_{A B}=13.5 \mathrm{~Hz}\right), 4.48,4.67\left(\mathrm{AB}, 2 \mathrm{H}, J_{A B}\right.$ $=8.1 \mathrm{~Hz}), \quad 5.37(\mathrm{~s}, 1 \mathrm{H}), 5.74(\mathrm{~s}, 1 \mathrm{H}), 6.17(\mathrm{~s}, 1 \mathrm{H}) ;{ }^{13} \mathrm{C} \mathrm{NMR}(75$ $\left.\mathrm{MHz}, \mathrm{CD}_{3} \mathrm{OD}\right) \delta 18.3,24.7,26.5,34.8,65.2,71.6,73.1,75.3,106.3$, 111.1, 127.8, 137.0, 159.5, 167.0; FT-IR (KBr) v 3329, 2930, 1764, $1627 \mathrm{~cm}^{-1}$; EI-MS (m/z, \%): 298 (37.60), 224 (66.93), 143 (59.02), 99 (63.17), 98 (43.55), 70 (30.80), 69 (70.00), 56 (100.00); Anal. Calcd for $\mathrm{C}_{14} \mathrm{H}_{19} \mathrm{O}_{7} \mathrm{~N}$ : C 53.67, H 6.11, N 4.47; Found C 53.94, H 6.46, N 4.63. 


\section{Supposed reaction transition state model.}

3-1. A possible reaction mechanism was presented as follows:
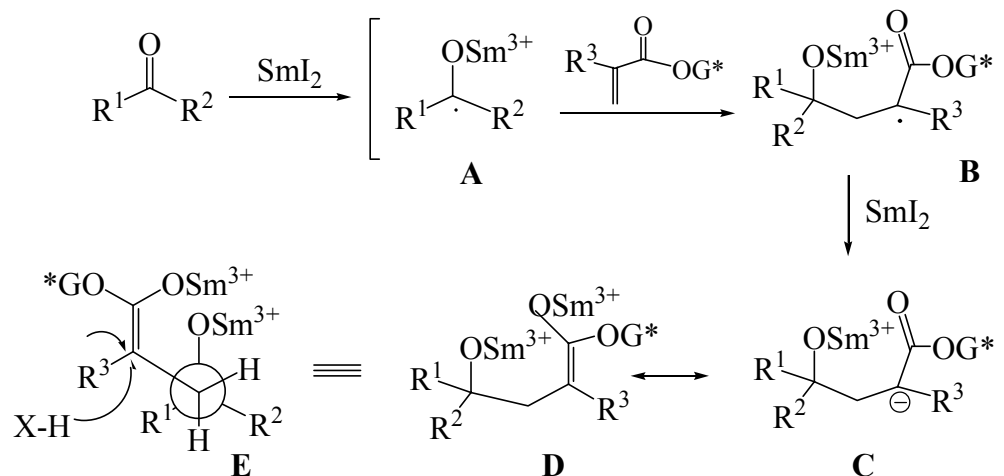

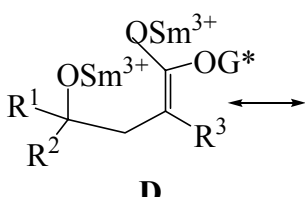<smiles>[R]C(CC([R])([R])[R])C(=O)O[Na]</smiles><smiles>[R]C(=CC([R])([R])O[Ga])C(=O)OCC</smiles>

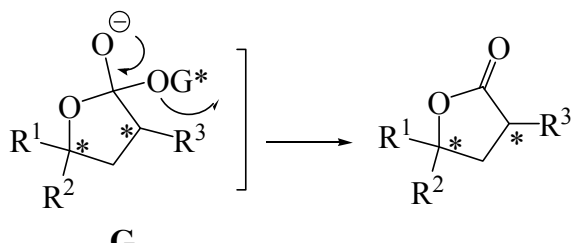

\section{Scheme 1}

\section{3-2. Supposed working models for the reactions with $\mathbf{3}$ and $\mathbf{4}$}

The plausible transition state $\mathbf{E}$ in Scheme 1 is the key for the explanation of protonation process in the reaction. The following are three supposed working models based on $\mathbf{E}$ to help understand the selectivity in these reactions. The exact transition state model and precise mechanistic explanation still remain unclear at this moment.

1) The reactions of using $\mathbf{3 a}, \mathbf{3 b}$ and $\mathbf{3} \mathbf{c}$ as substrates:

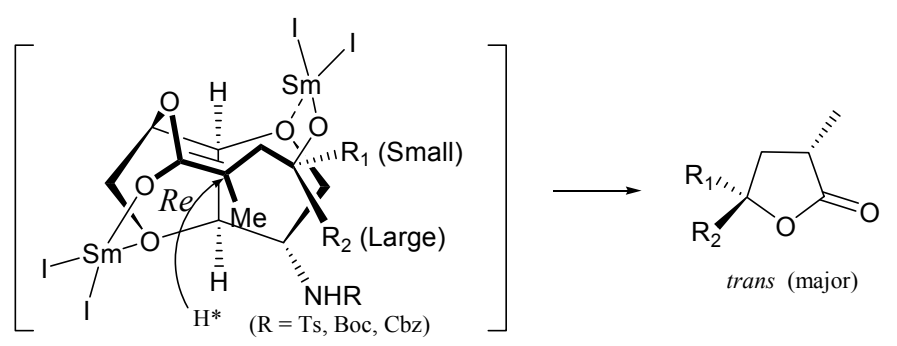

Figure 1 
2) The reactions of using $\mathbf{4 b}$ and $\mathbf{4 c}$ as substrates:

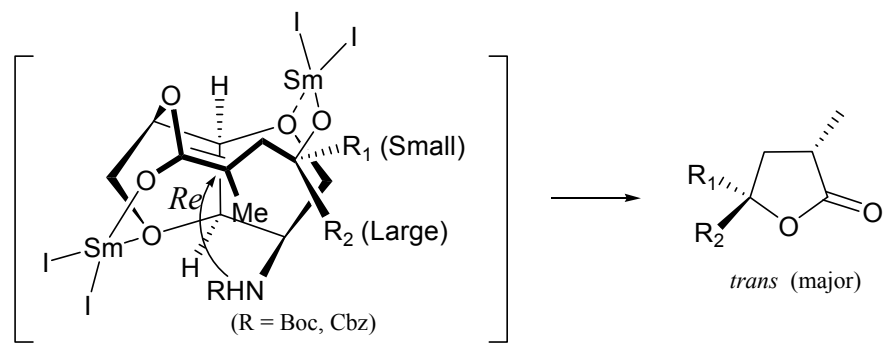

Figure 2

3) The reactions of using $\mathbf{4 a}, \mathbf{4 d}$ and $\mathbf{4 e}$ as substrates:

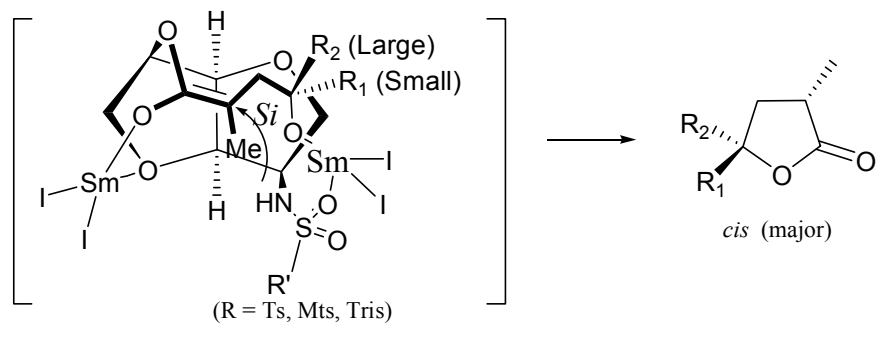

Figure 3 